\title{
Properties of the matter at high pressures
}

\author{
by V. P. Trubitsyn \\ Ricevuto il 7 dicembre 1960
}

The ionic crystal $\mathrm{MgO}$ is the most abundant crystal of the Earth's rocks. The state equation for $\mathrm{MgO}$ was obtained in this paper for pressures between zero and million atmospheres. The state equations for metal at high pressures are significant for physics of the Earth's core. In this paper these equation were obtained for metal in the analytical form.

Usual Thomas-Fermi-Dirak theory of the electron gas is applicable only at extremelly high pressures $p>10^{\circ}$ atm. Statistical GombasJensen theory of the ionic crystals and metals gives good results only at not high pressures. Therefore in this paper the modified statistical theory is used. By statistical theory the energy of some system of the electrons with density $\varrho$ is (Gombas 1949)

$$
\begin{gathered}
E(\varrho)=\int\left\{x_{k} \varrho^{5 / 3}+\left(V_{k}+1 / 2 V_{\varrho}\right) \varrho-x_{\alpha}{ }^{3} \varrho^{4 / 3}\right\} a \tau \\
x_{k}=2.871 \quad x_{a}{ }^{\prime}=0.8349
\end{gathered}
$$

It is the sum of kinetic (Fermi) and potential energy of the electrons in the field of the nuclear and of the exchange-correlation energy. If the electronic density $\varrho$ is known from the experiment and is substituted into the formula for the energy, then the problem leads only to integration.

In this paper the energy of the ionic crystal $\mathrm{MgO}$ is calculated. The electron density in crystal is taken as the sum of the overlapping electron densities of the ions. The electron density of the oxygen deformated in crystal was taken from Yamashita's and Koyima's works (1952). That of magnesium was taken according to Hartrec-Fock of isolated ions. For pressures $0-10^{8} \mathrm{~atm}$. the calculated energy (with account for the quantum correction) can be approximated by the formula (Trubitsyn 1958).

$$
E(v)=93.7 \exp \left(-1.69 v^{1 / 3}\right)-3.495 v^{-1 / 3}+0.582 \quad \frac{\text { at. unity }}{\text { atom }} .
$$


The pressure is found by the formula

$$
P=-\frac{d E}{d V}
$$

(1 at. unity of pressure $=3 \times 10^{8}$ atm., 1 at unity of volume $=\frac{0,0886}{\mu}$ $\frac{\mathrm{cm}^{3}}{5}, \mu-$ is the atomic number of the matter).

Figure 1 plots the calculated energy of the crystal $\mathrm{MgO}$ at $T=0^{\circ} \mathrm{K}$ (a) and the experimental energy of Bridgman (1949) extrapolated by Davydov's method (1956) (b).

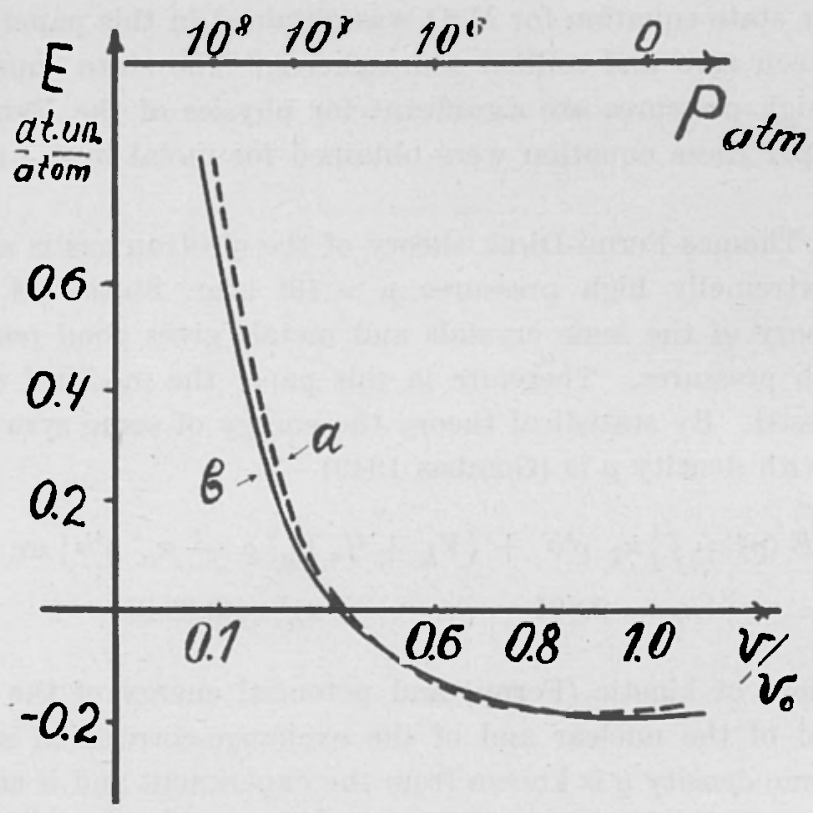

Fig. 1. - The energy of the ionic crystal MgO calculated by the author(curve $a$ ) and extrapolated from the experiment (curve $b$ ).

For metals the electron density in crystal is taken as the sum of the density of the ionic cores and that of valence electrons. The first was taken according to Hartrec-Fock for isolated ions and was approximated by the formula $o_{+}(r)=\operatorname{Aexp}(-b r)$. The second was approximated by the constant $\varrho_{v}(r)=q / V$ (Theis 1955). $q$ - is the number of valence electrons. The integrand was taken in form of power series 
and integrated. The dependence of energy and pressure upon volume was obtained for metals in the analytical form (Trubitsyn 1960)

$$
\begin{gathered}
E(v)=2.871 a^{0 / 3} v^{-2 / 3}-q^{4 / 3} v^{-1 / 3}\left[0.8349+1.4508 q^{2 / 3}\right] \\
+q^{5 / 3} b^{-3} v^{-5 / 3}\left[784.12-525.06 x+16.4934 x^{2}-12.026 x^{3}\right] \\
+q^{4 / 3} b^{-3} v^{-4 / 3}\left[720.26+244.81 x+31.517 x^{2}+3.4972 x^{3}\right] \\
+q b^{-3} v^{-1}\left[405.88 A^{2 / 3}-755.40 A^{1 / 3}-631.65 \mathrm{~A} \mathrm{~b}^{-2}\right] \\
x=\ln \left(A q^{-1} v\right) .
\end{gathered}
$$

The pressure is $P=-d E / d V$. Every metal was characterized by means of three parametres $A, b$ and $q$.

For magnesium $A-45, b=4,9$ and $q=2$. Then

$$
\begin{gathered}
p(v)=21.52 v^{-1}+6.095 v^{-5 / 3}-2.6352 v^{-4 / 3} \\
+v^{-7 / 3}\left[15.3236+5.6403 x+0.6753 x^{2}+0.09985 x^{3}\right] \\
+v^{-8 / 3}\left[49.4291-24.5025 x+1.7152 x^{2}-0.54083 x^{3}\right] \\
x=\ln (22.5 v) .
\end{gathered}
$$

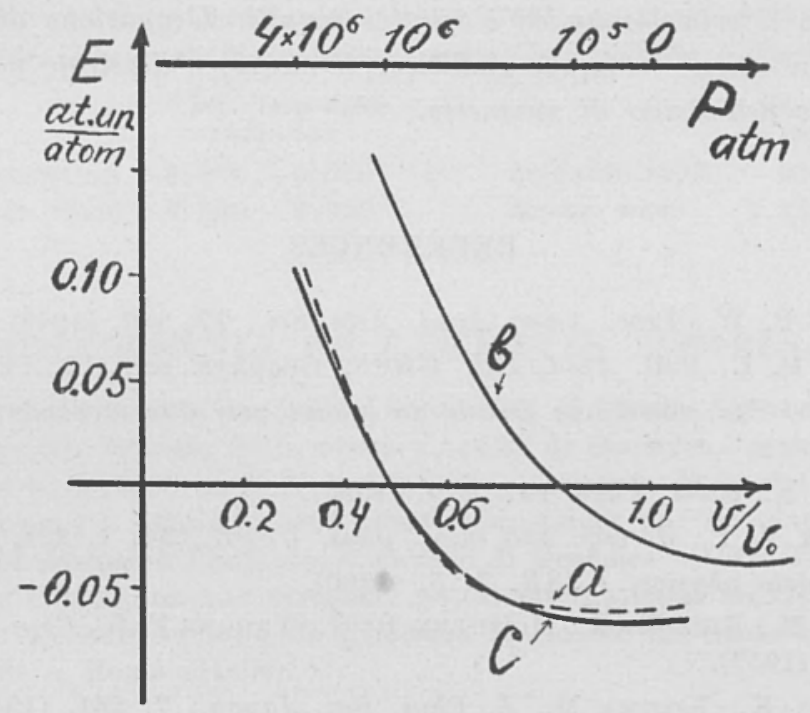

Fig. 2. - The energy of themetallic Mg caloulated from the author's formula (curve a) and extrapolated from the experiment (curve c), as well as calculated by Gombas (curve b). 
Figure 2 plots the energy and pressure of the metallic magnesium at $T=0^{\circ} \mathrm{K}$, calculated by the author $(a)$, this one calculated by Gombas (1949) (b) and the experimental energy and pressure of Walsh and others (1957), recounted for $T=0^{\circ} \mathrm{K}(c)$.

One can account for the temperature by the Davydov's method (1956).

It is a pleasure to thank Professor B. I. Davydov for helpful discussions.

\section{$S U M M A R Y$}

The modified statistical method is proposed for calculation the equation of state for ionic crystal and metals. The equation of state for metals is obtained in analytical form. The method is illustrated on magnesium and magnesium oxide.

\section{RIASSUNTO}

Si propone il metodo statistico modificato per il calcolo dell'equazione di stato per il cristallo ionico e relativi metalli. L'equazione di stato per $i$ metalli si ottiene in forma analitica. Il metodo e illustrato nel caso del magnesio e dell'ossido di magnesio.

\section{REFERENCES}

Bridgman P. W., Proc. Amer. Acad. Arts. Sci., 77, 187, (1949).

Davyoov B. I., Bull. Acad. Sci, URSS, Geophys. ser., 12, 1411, (1956).

Gombas P., Die statistische theorie des atoms und ihre anwendungen. Wien 1949.

Theis W. R., Zeits. Phys. 142, 511, (1955).

Trubitsyn V. P., J. exp. and theor. phys. USSR., 34, 1, 221, (1958).

- Solid state physics, USSR, 2, 5, (1960).

Walsh J. M., Rice M. H., McQueen R. G., Yarger F. L., Phys. Rev., 108, 196, (1957).

Yamashita K., KoJima M., J. Phys. Soc. Japan., 7, 261, (1952). 